\title{
Le pouvoir normatif de Google. Analyse de l'influence du moteur sur les pratiques des éditeurs
}

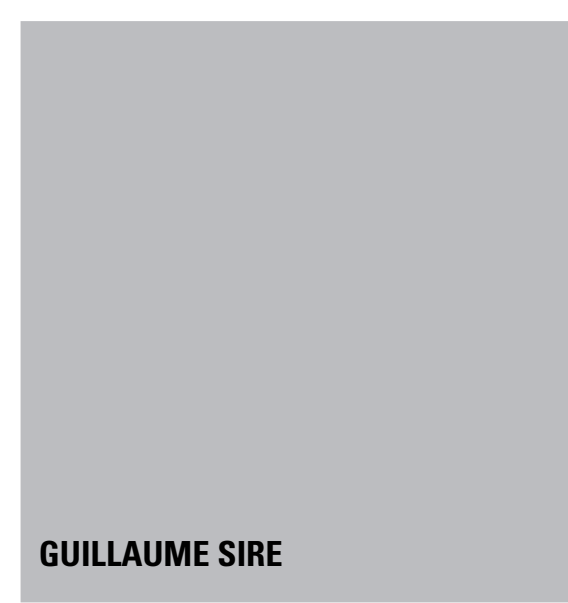

Dès lors qu'un éditeur souhaite que les documents qu'il a produits soient aussi visibles que possible, la méthode employée par le moteur Google pour hiérarchiser les informations est susceptible d'influencer ses pratiques. 0 r il se trouve que, paradoxalement, cette influence ne va pas toujours dans le sens des intérêts de l'entreprise Google. Nous expliquons pourquoi, avant d'expliquer comment les concepteurs du moteur essayent d'orienter l'influence qu'ils exercent sur la production éditoriale dans un sens qui leur est favorable en publiant des recommandations destinées aux éditeurs et en menaçant de sanctionner les comportements jugés malveillants. Finalement les effets normatifs résultent d'une tension entre l'algorithme, le discours et une règle éthique, et ils n'influencent pas seulement les contenus mais aussi le moteur lui-même.

Mots-clés. Web, Internet, Google, PageRank, lien hypertexte, SEO, moteur de recherche, algorithme, gouvernementalité
1. Sur le terme machine, voir : Dominique Cardon, «Dans l'esprit du PageRank. Une enquête sur l'algorithme de Google ", Réseaux, 177, 2013, p. 90-91.

2. À propos du répertoire d'actions possibles, voir : Guillaume Sire, Bernhard Rieder, "Dans les ramures de l'arbre hypertexte, Analyse des incitations générées par l'opacité du moteur Google ", French Journal for Media Research, 3, 2015, [en ligne] http://frenchjournalformediaresearch.com/lodel/docannexe/file/468/ arbre_de_navigation.pdf, consulté le 15 février 2016. 
Cette interaction n'est pas isotopique ${ }^{3}$ : la liste qui apparaît à l'écran est le résultat de différentes actions effectuées en différents lieux. Elle n'est pas synchronique : les actions dont la production de la liste découle ne sont pas effectuées au même moment. Elle n'est pas non plus synoptique : seules quelques entités, jamais toutes, apparaissent à un moment donné. Elle n'est pas homogène : les relais qui assurent le déroulement de l'interaction n'ont pas la même matérialité. Enfin, elle n'est pas isobarique : les actants ${ }^{4}$ n'ont pas la même capacité d'influence, le même pouvoir, la même force ${ }^{5}$.

Considéré comme le lieu d'une telle interaction, le moteur Google ne peut pas être présenté sous les traits d'une machine neutre, non humaine, un monstre inorganique et objectif. Il n'y a pas de moteur sans entreprise, et sans les salariés de cette entreprise, les dirigeants, les actionnaires, pas plus qu'il n'y aurait de moteur s'il n'y avait aucun éditeur ni aucun utilisateur. Il n'y a pas de machine sans que des actions soient coordonnées, interprétées, traduites, ni sans négociations, sans choix empiriques. Et il est, en outre, impensable de concevoir un dispositif censé hiérarchiser des documents par ordre de pertinence sans que soient confrontées des postures épistémologiques, cela car aucun procédé dont l'information est l'objet ne peut échapper à la confrontation des subjectivités. Finalement, le moteur ne peut pas être considéré indépendamment de l'action humaine, des représentations et de l'écosystème dans lequel il agit et fait interagir. S'il existe une machine, c'est qu'il y a des machinistes et une machination.

En scrutant le dispositif de communication ${ }^{6}$ et cette machination dont le moteur est à la fois l'objet et le sujet, l'effet et la cause, on s'aperçoit que Google est une machine à faire (le moteur produit), une machine à faire voir (il montre),

3. Nous reprenons ici les caractéristiques de l'interaction (non isotopique, non synchronique, non synoptique, non homogène et non isobarique) définie par: Bruno Latour, Changer de société, refaire de la sociologie, Paris, La Découverte, 2007, p. 294.

4. Le concept d' «actant» est importé de la sémiotique en sociologie des techniques par les théoriciens de l'acteur-réseau, cf. Madeleine Akrich, Michel Callon \& Bruno Latour, Sociologie de la traduction. Textes fondateurs, Paris, Presses des Mines de Paris, 2006, 304 p. Il désigne tout ce qui peut prendre part à une interaction, que cela soit humain ou non-humain. Autrement dit, les choses et les concepts peuvent être des actants. Cela permet « d'élargir la question sociale à tous les êtres qui interagissent dans une association et qui s'échangent leurs propriétés ", cf. : Bruno Latour, "Une sociologie sans objet ? Remarques sur l'interobjectivité » in Octave Debary \& Laurier Turgeon, Objets et mémoires, Paris, Maison des Sciences de l'Homme, 2007b, p. 51. Cette distinction acteurs/actants est utile ici, car nous verrons avec l'analyse du PageRank comment une formule mathématique peut faire et faire faire des actions qui ne sont pas forcément celles que souhaiteraient faire et faire faire les auteurs de ladite formule.

5. Pour plus de détails concernant les caractéristiques de l'interaction qui prévaut à la production de l'appariement requête-résultat, voir : Bernhard Rieder, Guillaume Sire, "Conflicts of Interest and Incentives to Bias: A Microeconomic Critique of Google's Tangled Position on the Web », New Media \& Society, 16, 2014, p. 195-211.

6. Sur ce terme, voir : Laurence Monnoyer-Smith, « Le Web comme dispositif : comment appréhender le complexe ? " in Christine Barats (dir.), Manuel d'analyse du Web en Sciences Humaines et Sociales, Paris, Armand Colin, 2013, 258 p. Voir aussi : Violaine Appel, Hélène Boulanger \& Luc Massou, Les dispositifs d'information et de communication. Concepts, usages et objets, Bruxelles, De Boeck, 2010, 246 p. 
une machine à faire dire (il propose) et une machine à faire faire ${ }^{7}$ : non seulement il ouvre des chemins de navigation hypertexte, mais, de plus, il suscite certaines actions et réactions de la part de ses utilisateurs et de la part, aussi, des éditeurs de contenus. Comment ne pas imaginer qu'un moteur de recherche concentrant plus de $90 \%$ des parts de marché ${ }^{8}$, et par conséquent susceptible d'apporter un trafic considérable aux éditeurs, influence la manière dont ces derniers prennent la parole sur le Web et donc la parole elle-même?

Il semble évident que certains éditeurs, si ce n'est tous les éditeurs, agissent en fonction de ce qu'ils ont intérêt à faire pour figurer en haut des classements. Dès lors, il convient de se demander ce que les éditeurs savent à propos de ce qu'il leur faudrait faire et comment cela, exactement, peut influencer - et même normaliser - leurs actions.

Google publie des recommandations sur son centre d'aide, dont l'objet est justement de prévenir les éditeurs de ce qu'ils doivent faire pour maximiser leur exposition. Theo Röhle considère qu'il s'agit d'une façon d'imposer « une norme de publication » en mettant en place un " régime disciplinaire ${ }^{9}$. Il se réfere aux travaux de Michel Foucault, d'après qui le pouvoir s'exerce davantage qu'il ne se possède, et circule à travers « un faisceau plus ou moins organisé, plus ou moins pyramidalisé, plus ou moins coordonné, de relations » ${ }^{10}$, de telle sorte que les effets normatifs découlent d'un assemblage entre prescription et constitution ${ }^{11}$. Dans le cas du moteur Google, cet assemblage et les tensions dont il est à la fois l'objet et le sujet, ainsi que les effets normatifs qui en découlent en matière de pratiques de publication, sont caractéristiques de ce qui se passe sur Internet, où les dynamiques normatives résultent d'une multitude de médiations au sein d'un espace que pour cette raison Françoise Massit-Folléa, Cécile Méadel et Laurence Monnoyer-Smith qualifient de « multi-normatif ${ }^{12}$.

En observant nous-mêmes les tenants et les aboutissants du faire-faire évoqué par Theo Röhle, ainsi que leurs effets normatifs, nous avons remarqué, paradoxalement, que le résultat de l'influence exercée par Google sur les pratiques

7. Sur le dispositif conçu comme machine à faire, à faire voir, à faire dire et à faire faire, voir : Julia Bonaccorsi, Virginie Julliard, "Dispositifs de communication numeriques et mediation du politique. Le cas du site Web d'Ideal-Eu ", in Mona Aghababaie, Audrey Bonjour, Adeline Clerc \& Guillaume Rauscher (dir.), Usages et enjeux des dispositifs de mediation, Nancy, Presses universitaires de Nancy, 2010, p. 65-78. Voir aussi : Clément Mabi, "Les conceptions de la participation citoyenne inscrites au sein d'un dispositif de concertation ", Journées doctorales GIS Participation, 2011, [en ligne] http://www.participation-et-democratie.fr/content/les-conceptions-dela-participation-citoyenne-inscrites-au-sein-dun-dispositif-de-concertati, consulté le 15 février 2016.

8. AT Internet, Baromètre des moteurs de recherche, 2014, [en ligne] http://www.atinternet.com/ressources/ressource/barometre-des-moteurs-de-recherche-novembre2014/, consulté le 15 février 2016.

9. Theo Röhle, «Dissecting the Gatekeepers. Relational Perspectives on the Power of Search Engines ", in Konrad Becker \& Felix Stalder (dir.) Deep Search. The Politics of Search beyond Google, Innsbruck, StudienVerlag, 2009, p. 117-132.

10. Michel Foucault, « Le jeu de Michel Foucault », Ornicar?, 10, 1977, p. 66.

11. Michel Foucault, Surveiller et Punir, Paris, Gallimard, 1975, p. 35.

12. Françoise Massit-Folléa, Cécile Méadel \& Laurence Monnoyer-Smith, Normative Experience in Internet Politics, Paris, Presses des Mines, $266 \mathrm{p}$. 
de publication n'était pas forcément favorable à l'entreprise Google, et pouvait même nuire à ses intérêts. Il semblerait par conséquent que Google soit obligé d'agir pour orienter la dynamique de normalisation dans un sens qui lui convient. Un processus de négociation se met alors en place entre des acteurs qui, chacun à son niveau, essayent d'influencer l'action et les paramètres du dispositif.

Dans le but de compléter et de préciser la réflexion amorcée par Theo Röhle, nous avons analysé les tenants de ce processus de négociation en concentrant notre focale sur le PageRank, algorithme fondateur du moteur de recherche Google, dont nous avons considéré les dimensions performatives : ce qu'il fait, ce qu'il dit et ce que cela fait dire et faire aux acteurs concernés par ce faire et ce dire.

\section{L'ALGORITHME DU MOTEUR GOOGLE}

Le PageRank est un algorithme dont le principe est de considérer à la fois que chaque lien hypertexte pointant vers une page est un vote pour cette page et que plus une page reçoit de votes, plus elle est pertinente, et plus son vote, lorsqu'elle pointe elle-même vers d'autres pages, donne de la valeur à la page pointée ; il s'agit également de considérer que lorsqu'une page contient, par exemple, six liens vers d'autres pages, chacun de ces liens sera porteur de deux fois moins d'autorité que si la page en contenait seulement trois. Couplé avec des procédés d'analyse statistique des textes, cet algorithme permet à Google d'intégrer la topographie hypertexte au calcul de pertinence en identifiant le degré de centralité des contenus sur le Web ${ }^{13}$ - c'est-à-dire une page vers laquelle de nombreux liens pointent est considérée comme centrale dans ladite topographie, une page vers laquelle très peu de liens pointent y est marginale, enfin une page vers laquelle ne pointe aucun lien en est exclue.

PageRank a été la clef de voûte du moteur Google, qui grâce à lui s'est démarqué d'une concurrence florissante à la fin des années $1990^{14}$. Puisqu'il trie et hiérarchise l'information, on peut dire de cet algorithme qu'il a des dimensions à la fois politique $^{15}$ et morale ${ }^{16}$. De nombreuses critiques lui sont adressées, notamment en raison de sa tendance à avantager les pages Web déjà les plus visibles ${ }^{17}$. Quand un contenu est bien placé dans les classements du moteur Google, il attire des visiteurs, ce qui maximise ses chances d'attirer des liens, et ce qui renforcera sa visibilité dans les listes de résultats en renforçant sa centralité topographique. Finalement,

13. Pour une analyse détaillée du PageRank, voir : Bernhard Rieder, « What is in PageRank? A Historical and Conceptual Investigation of a Recursive Status Index », Computational Culture, 2012, [en ligne] http://computationalculture.net/article/what_is_in_pagerank, consulté le 15 février 2016.

14. John Battelle, The search: how Google and its rivals rewrote the rules of business and transformed our culture, Nicholas Brealey Publishing, 2005, 288 p. Voir aussi : Elizabeth Van Couvering, "The History of the Internet Search Engine: Navigational Media and the Traffic Commodity », in Michael Zimmer \& Amanda Spink (dir), Web Search. Multidisciplinary Perspectives, Berlin, Springer, 2008, p. 177-206.

15. Lucas D. Introna, Helen Nissenbaum, "Shaping the Web: why the politics of search engines matters ", The Information Society, 16, 2000, p. 169-185.

16. Dominique Cardon, 2013, « Dans l’esprit du PageRank. », art. cit.

17. Matthew Hindman, Kostas Tsioutsiouliklis \& Judy A. Johnson, « "Googlearchy”: How a Few Heavily Linked Sites Dominate Politics on the Web ", Annual meeting of the Midwest Political Science Association, 2003 
il semblerait que $90 \%$ de l'autorité PageRank soit concentrée sur $10 \%$ des sites $\mathrm{Web}^{18}$.

Le PageRank est connu dans le détail, ayant fait l'objet de deux articles scientifiques ${ }^{19}$ et de deux dépôts de brevets rédigés sous la houlette des fondateurs de Google ${ }^{20}$. C'est la seule partie connue de l'algorithme. Depuis la mise en ligne du dispositif, en 1998, de nombreux critères ont été ajoutés à propos desquels nous n'avons pas de détails précis ${ }^{21}$. Même si des suppositions existent, il est extrêmement difficile de vérifier avec certitude que tel ou tel critère est pris en compte. L'algorithme (dont PageRank n'est désormais qu'un sous-ensemble) est devenu un secret industriel. Cela est essentiellement justifié par deux raisons : 1. empêcher les stratégies d'imitation de la part des moteurs concurrents ; 2 . décourager les éditeurs qui risqueraient, dans le cas où ils en sauraient trop à propos des critères permettant d'évaluer la pertinence d'un document, d'intervenir dans le but d'orienter systématiquement les résultats en leur faveur. Cette absence de transparence crée une controverse d'ordre épistémologique : Google propose de répondre à toutes les questions imaginables pourvu qu'aucun internaute ne lui demande comment son moteur parvient à réaliser un tel tour de force. Comme dit le philosophe Paul Mathias, «c'est un peu comme si savoir exigeait qu'on ne sût pas pourquoi ni comment l'on sait $»^{22}$.

\section{LE faire-faire DU faire}

Étant donné ce qu'ils savent du PageRank, les éditeurs désireux de maximiser la visibilité de leurs documents dans les listes de résultats générées par Google sont incités à effectuer des liens vers leurs propres pages, de façon à s'auto-distribuer leur autorité plutôt que la diluer en pointant vers l'extérieur. Cette stratégie consistant à canaliser le flux hypertexte dans le but d'optimiser le référencement des pages s'appelle le PageRank sculpting. Elle peut conduire à créer des bulles de contenus, qui agissent alors comme des culs-de-sac topographiques : des liens vont vers ces bulles, mais aucun, ou très peu, n'en ressort. On observe un tel égocentrisme à maints endroits sur le Web. Nous avons nous-même effectué une observation de ce phénomène, en 2012 puis en 2015, au sein de six sites français

18. Gopal Pandurangan, Prabhakar Raghavan \& Eli Upfal, « Using PageRank to characterize Web structure ", Internet Mathematics, 3, 2006, p. 1-20, cit. in Dominique Cardon, 2013, " Dans l'esprit du PageRank. ", art. cit.

19. Sergey Brin, Larry Page, "The Anatomy of a Large-Scale Hypertextual Web Search Engine ", Computer Science Department, Stanford University, 1998 ; Larry Page, Sergey Brin, Rajeev Motwani \& Terry Winograd, « The PageRank Citation Ranking: Bringing Order to the Web », Technical Report, Stanford InfoLab, 1999.

20. Brevet $\mathrm{n}^{\circ} 1$ : Larry Page, "Method for Node Ranking in a Hyperlinked Database ", US Patent 6,285,999, filed January 9, 1998, and issued September 4, 2001 ; Brevet $n^{\circ} 2$ : Larry Page, « Method for Scoring Documents in a Linked Database ", US Patent 6,799,176, filed July 6, 2001, and issued September 28, 2004.

21. Raphaël Richard, Google décodé : les 300 critères de classement $t$. 1 \&2, Webmarketing Academy, 2011. Voir aussi : Olivier Andrieu, Reussir son referencement Web. Strategie et techniques SEO, Eyrolles, 2013.

22. Paul Mathias, Qu'est-ce que l’Internet?, Paris, Vrin, coll. «Chemins Philosophiques », 2009, p. 39-41. 
Tableau 1. L'égocentrisme hypertexte ${ }^{23}$

\begin{tabular}{|c|c|c|c|c|c|c|}
\hline & \multicolumn{2}{|c|}{$\begin{array}{l}\text { Nombre de liens dans } \\
\text { le corps de l'article }\end{array}$} & \multicolumn{2}{|c|}{$\begin{array}{l}\text { Nombre liens vers une page } \\
\text { du même site }\end{array}$} & \multicolumn{2}{|c|}{$\begin{array}{c}\text { Nombre de liens vers un } \\
\text { autre site }\end{array}$} \\
\hline & 2012 & 2015 & 2012 & 2015 & 2012 & 2015 \\
\hline Lexpress.fr & 315 & 249 & $284(90 \%)$ & $196(78 \%)$ & $31(10 \%)$ & $53(22 \%)$ \\
\hline Lefigaro.fr & 155 & 113 & $133(86 \%)$ & $90(80 \%)$ & $22(14 \%)$ & $23(20 \%)$ \\
\hline Lemonde.fr & 1258 & 708 & $1202(96 \%)$ & $698(99 \%)$ & $56(4 \%)$ & $10(1 \%)$ \\
\hline Nouvelobs.com & 128 & 251 & $114(89 \%)$ & $171(68 \%)$ & $14(11 \%)$ & $80(32 \%)$ \\
\hline Leparisien.fr & 188 & 226 & $177(94 \%)$ & $197(87 \%)$ & $11(6 \%)$ & $29(13 \%)$ \\
\hline Lepoint.fr & 73 & 153 & $70(96 \%)$ & $146(95 \%)$ & $3(4 \%)$ & $7(5 \%)$ \\
\hline TOTAL & 2117 & 1700 & 1980(94\%) & $1498(88 \%)$ & $137(6 \%)$ & $215(12 \%)$ \\
\hline
\end{tabular}

de presse en ligne, dont les résultats montrent clairement que les sites observés ont tendance à pointer vers leurs propres pages ( $c f$. tableau 1$)$.

Cette incitation à l'égocentrisme hypertexte est problématique pour les concepteurs du moteur comme pour les éditeurs et les internautes, car si tous les éditeurs pointaient vers leurs propres contenus, le PageRank ne remplirait plus la mission qui consiste à hiérarchiser les contenus par ordre de pertinence. Pire, le moteur ne pourrait plus fonctionner, car pour découvrir les pages existantes sur le Web, Google a besoin de faire naviguer des logiciels appelés crawlers de lien en lien ${ }^{24}$, lesquels ne pourraient plus mener à bien la procédure d'indexation s'il n'y avait pas de liens permettant de passer d'un site à un autre. Autrement dit, le PageRank porte en lui sa propre négation : l'algorithme incite les éditeurs à entreprendre certaines actions risquant de l'empêcher de faire ce pour quoi il a été inventé. Il existe une tension entre le faire et le faire-faire du PageRank.

Finalement, il apparaît que le PageRank est un objet dont l'action ne dépend pas seulement de ce qu'ont intérêt à faire et faire faire ses concepteurs, mais aussi de ce que l'algorithme fait et fait faire par lui-même. Le PageRank, considéré comme un actant à part entière, n’est pas simplement un énoncé mais également un énonçant, il n'est pas seulement déterminé mais aussi déterminant.

23. Résultat d'une étude menée deux fois, en 2012 et 2015. Nous avons sélectionné six sites Web adossés à des titres de presse imprimée nationaux d'information politique et générale (quotidiens ou magazines) : Lemonde.fr, Lefigaro.fr, Lepoint.fr, Lexpress.fr, Leparisien.fr, Nouvelobs.com. Pour chacun de ces sites, nous avons prélevé les trois articles qui se trouvaient en tête de la page d'accueil au moment où nous les visitions (en fin d'après-midi), durant quatorze jours consécutifs en octobre 2012, pour un total de 252 articles. Nous avons publié les résultats de cette première étude dans : Guillaume Sire, Google, la presse et les journalistes. Analyse interdisciplinaire d'une situation de coopétition, Bruxelles, Institut du Droit de la Concurrence/Bruylant, coll. « Sciences Politiques », 2015, 426 p. Puis nous avons réitéré l'observation, de la même façon exactement, en septembre 2015. Les résultats détaillés de cette deuxième étude, analysés au regard des résultats de 2012, seront publiés dans un ouvrage collectif à venir, dont le nom sera Le Web dans les rédactions, 20 après, dirigé par Jean-Baptiste Legavre et Rémy Rieffel. Le titre du chapitre sera : «Tout ça pour ça ? Titres fonctionnels et égocentrisme hypertextuel ». 24. Pour le détail du fonctionnement, voir : Guillaume Sire, Les moteurs de recherche, Paris, La Découverte, coll. « Repères », 2016, 128 p. 


\section{LE faire-faire DU dire}

Google donne des recommandations aux éditeurs par l'intermédiaire de son site Web appelé « centre de support ${ }^{25}$, de différents blogs officiels sur lesquels sont données des informations concernant le moteur ${ }^{26}$ et d'interventions régulières de ses porte-parole dans la presse spécialisée et sur leurs comptes Youtube. Il s'agit d'un dire : ce n'est pas directement le moteur cette fois qui suggère d'entreprendre certaines actions, mais les employés de la firme qui rédigent des textes et réalisent des vidéos pour expliquer aux éditeurs ce qu'il convient de faire dans le cas où ils souhaiteraient recevoir un trafic substantiel sur leurs pages. Google prévient notamment que : «Les webmasters qui s'efforcent de respecter ces principes de base offriront aux internautes une navigation plus agréable et obtiendront un meilleur classement que ceux qui recherchent en permanence les failles à exploiter ${ }^{27}$.

Google est dans l'impossibilité de garantir à un éditeur qui se conformerait à de telles recommandations qu'il obtiendra effectivement un trafic substantiel. Cette impossibilité vient du fait que si le succès était promis à tous les éditeurs ayant scrupuleusement respecté les règles ainsi édictées, le moteur ne pourrait plus hiérarchiser les contenus par ordre de pertinence.

Google prévient également que certaines actions sont considérées comme « illicites » et qu'elles peuvent être "susceptibles d'entraîner l'application d'une action manuelle pour cause de spam, une sanction de type algorithmique ou le retrait définitif des sites concernés de l'index ${ }^{28}$. Le recours au terme "illicite » suggère que Google édicte une norme de comportement ${ }^{29}$. Les bénéfices de celui qui se conformera à cette norme sont hypothétiques, tandis que les risques de sanction, eux, sont bien réels, clairement affichés. Google punira les éditeurs qui auront agi de façon anormale, et récompensera peut-être ceux qui auront agi normalement.

Parmi les actions illicites, Google demande aux éditeurs de s'abstenir de participer à des systèmes de liens. Il s'agit clairement d'enrayer le faire-faire du faire décrit ci-avant en décourageant les pratiques de PageRank Sculpting. Les systèmes de liens sont désignés comme suit ${ }^{30}$ :

Achat ou vente de liens pour améliorer le classement PageRank. Cela inclut l'achat de liens ou de messages contenant des liens, l'échange de biens ou de services contre

25. Google, " Consignes aux webmasters ", Aide Search Console, [en ligne] https://support.google.com/webmasters/answer/35769?hl=fr\&ref_topic=6002025, consulté le 15 février 2016.

26. Par exemple : https://googleblog.blogspot.fr/ ; ou encore : http://googlewebmastercentralfr.blogspot.fr/, pages consultées le 15 février 2016.

27. Google, « Consignes aux webmasters », [en ligne], https://support.google.com/webmasters/answer/ 35769 ?hl=fr\&ref_topic=6002025, consulté le 15 février 2016.

28. Ibid. Voir aussi l'article de Brigitte Simonnot au sein du présent dossier.

29. Une action illégale enfreint la loi, alors qu'une action illicite est une action qui enfreint une norme de comportement, laquelle peut être juridique et/ou morale.

30. Google, " Systèmes de liens ", Aide Search Console, [en ligne] https://support.google.com/webmasters/answer/66356, consulté le 15 février 2016. 
des liens, ou encore l'envoi d'un produit « gratuit » en échange d'un commentaire incluant un lien.

Échanges de liens excessifs ("Établissez un lien vers mon site et j'établirai un lien vers le vôtre ») ou pages partenaires utilisées exclusivement à des fins d'échange de liens.

Vastes campagnes de marketing via des articles ou de publication de messages de blog en tant qu'invité, avec des liens de texte d'ancrage riches en mots-clés.

Utilisation de programmes ou services automatisés pour créer des liens vers votre site.

En plus de cette recommandation, Google a également prévenu les éditeurs que les liens pointant vers l'extérieur étaient récompensés ${ }^{31}$ et que les liens vers soi-même étaient moins porteurs d'autorité PageRank que les liens provenant de l'extérieur ${ }^{32}$. Il y a ici deux incitations contraires, étant donné ce qui est dit aux éditeurs par les porte-parole de Google et ce que les éditeurs savent du fonctionnement du PageRank. Google essaye d'utiliser le faire-faire de son dire pour contrebalancer les effets du faire-faire de son faire.

Le détail de l'algorithme n'étant jamais dévoilé, les éditeurs n'ont pas d'autre choix que de croire sur parole les représentants de Google quand il leur est annoncé que le respect de telle ou telle consigne leur permettra de recevoir davantage de trafic sur leurs pages. C'est ce tout indémêlable entre algorithme et discours à propos de l'algorithme qui génère des effets normatifs, lesquels ne seront pas forcément ceux que les concepteurs du moteur auraient souhaités.

Remarquons que si aucun éditeur ne connaissait l'existence de Google, ou bien encore si le référencement sur le moteur ne constituait pour aucun éditeur un élément d'importance stratégique, les concepteurs pourraient faire fonctionner le dispositif sans que cela n'ait d'influence sur le nombre de liens faits vers l'extérieur. Les effets normatifs disparaîtraient.

\section{ChapeauX noIrs et ChapeauX blancs}

Il est d'usage de différencier parmi les éditeurs ceux qui ont un comportement ne visant pas à tromper le calcul de pertinence effectué par le moteur de recherche Google, appelés white hats (chapeaux blancs), et ceux qui au contraire tentent de manipuler les résultats et de tromper l'algorithme, appelés black hats (chapeaux noirs).

31. Olivier Andrieu, "Google analyse-t-il les liens sortants des pages ? ", Abondance, 7 juin 2011, [en ligne] http://www.abondance.com/actualites/20110607-10920-google-analyse-t-il-les-lienssortants-des-pages.html, consulté le 15 février 2016.

32. Susan Moskwa "Reorganizing internal vs. external backlinks », Google Webmaster Central Blog, 31 août 2011, [en ligne] http://googlewebmastercentral.blogspot.fr/2011/08/reorganizing-internal-vsexternal.html, consulté le 15 février 2016. 


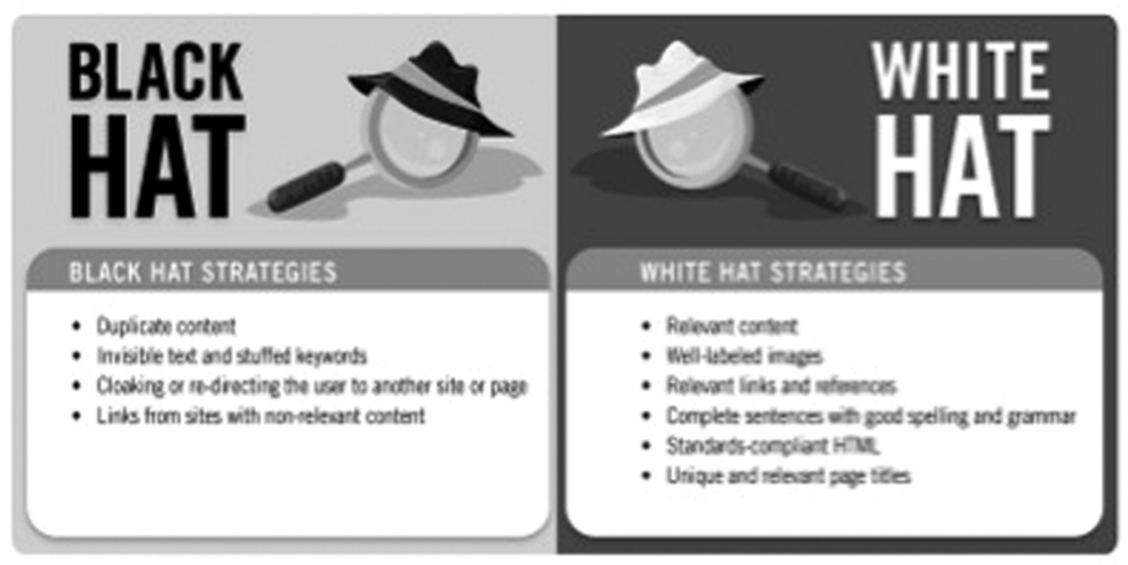

Figure 1. Chapeaux blancs et chapeaux noirs (Prolific Web solutions) ${ }^{33}$

Une telle dichotomie suppose qu'il y ait d'un côté un comportement éthique, c'est-à-dire une "pratique réfléchie de la liberté " ${ }^{34}$ qui ne met pas en péril les intérêts des différents acteurs concernés par l'action du dispositif, et de l'autre un comportement malveillant, non éthique, qui, s'il se généralisait, aboutirait à une diminution de la qualité du moteur et pourrait même le rendre inopérant.

La frontière entre chapeau blanc et chapeau noir est inévitablement travaillée par la tension que nous avons décrite entre le dire et le faire (faire ce que Google dit de faire ou ce que les actions de Google suggèrent de faire). Comme souvent en matière d'éthique, la limite n'est pas évidente à situer entre un comportement acceptable et un comportement qui ne le serait pas. Le black hat change notamment de définition selon le point de vue qu'on adopte.

Pour la société Google et les référenceurs, ce terme recouvre l'ensemble des procédés qui biaisent les algorithmes de moteurs de recherche. Pour les autres, il rejoint celui de nuisance, de spamming, de messages et commentaires non sollicités sur leurs supports, [...] Pratiquement, on remarque que le terme englobe un large panel de pratiques qui pour la plupart visent au profit économique. Ainsi, sur les forums dédiés au black hat $S E O$, se mêlent les personnes souhaitant favoriser l'indexation de leur site internet par tous moyens, mais aussi des individus cherchant le bénéfice par les manières les plus illégales qui soient, tels que le piratage de sites internet, de cartes de crédit, aussi, il n'est pas étonnant que cette activité ait mauvaise presse. ${ }^{35}$

Il existe une tension entre le caractère individuel de l'action et son caractère collectif, à partir de laquelle il est possible de déterminer une éthique éditoriale qui sera la même quel que soit le point de vue adopté. Pour décrire cette éthique,

33. Visuel repris sur Prolific Web Solutions, [en ligne] http://www.prolificwebsolutions.com, consulté le 15 février 2016.

34. Michel Foucault, Dits et écrits, t. 2, Paris, Gallimard, 1994, p. 1530.

35. Charles-Victor Boutet, Samy Ben Amor, « Vers l'active SEO 2.0 », Les Cahiers du numérique, 6, 2010, p. 179-198. 
considérons à nouveau l'influence de Google sur l'usage des liens hypertextes. Les logiciels de crawling du moteur ayant besoin des liens pour prendre connaissance des documents disponibles sur le Web, si aucun éditeur n'effectuait de liens vers l'extérieur, cela ne servirait plus à rien de ne pas en faire puisque le moteur ne pourrait plus fonctionner. En n'effectuant des liens que vers ses propres contenus, un éditeur fait donc précisément ce que ses homologues ne doivent pas faire pour que sa propre action puisse porter ses fruits. Autrement dit, son action n'aura l'effet escompté que si les autres éditeurs agissent de manière inverse à la sienne. Une telle pratique black hat peut être considérée comme étant non éthique, non seulement au regard des règles fixées par le discours de Google, mais au sens de Kant, dans la mesure où elle ne répond pas à l'impératif catégorique : le principe qui prévaut ne peut pas être généralisé à tous les éditeurs, car s'il l'était la pratique elle-même deviendrait inopérante et donc, injustifiée. En suivant ce principe, les actions éthiques seraient celles qui pourraient être effectuées par tous les éditeurs sans mettre en péril le fonctionnement ou l'existence du dispositif. C'est à notre avis la meilleure définition possible des pratiques white hat, dans la mesure où elle ne varie pas en fonction des points de vue.

Cette mise en tension entre une pratique de publication acceptable et une pratique inacceptable du point de vue éthique - c'est-à-dire entre des comportements visant l'intérêt collectif (ou ne s'y opposant pas) et des comportements visant le seul intérêt individuel au détriment de l'intérêt de tous les autres - a des effets concrets sur la dynamique normative du dispositif, dans la mesure où, comme nous nous apprêtons à l'expliquer, elle conduit les concepteurs du moteur Google à modifier l'algorithme et donc, en quelque sorte, à changer les règles du jeu auxquels se soumettent les white hats sous prétexte que des black hats les auraient enfreintes ou contournées.

\section{TOURS ET DÉTOURS DU DISPOSITIF}

Les concepteurs du moteur Google re-paramètrent régulièrement l'algorithme pour réussir à sanctionner les black hats, ou en tout cas pour diminuer la portée de leurs actions et diminuer aussi la force des incitations à entreprendre ces actions. Dans le cas des incitations à l'égocentrisme hypertexte induites par le PageRank et décrites ci-avant, il s'agira de modifier l'algorithme de façon à identifier les systèmes de liens et à défavoriser leurs auteurs dans les listes de résultats.

Un aller-retour a lieu entre ceux qui produisent l'énoncé (les contenus) et ceux qui fabriquent la machine à produire le méta-énoncé (les liens menant vers les contenus). Les éditeurs sont influencés dans leurs pratiques éditoriales, certes, c'est ce qu'a suggéré Theo Röhle ${ }^{36}$, mais il faut également considérer que les concepteurs du moteur Google doivent eux aussi accepter de procéder à des changements ${ }^{37}$ et subir ainsi l'influence des pratiques black hats : le faire-faire a lieu dans les deux

36. Theo Röhle, 2009, « Dissecting the Gatekeepers. » art. cit.

37. Sur la différence entre le stratège et le tacticien, voir : Michel De Certeau, L'invention du quotidien, 1. Arts de faire, Paris, UGE/10-18, 1980, 348 p. 


\section{Questionner les pouvoirs éditoriaux de Google

sens. Cet aller-retour donne lieu à de multiples modifications algorithmiques ${ }^{38}$, dont la vocation est toujours d'atténuer la voix des black hats et de récompenser les white hats. Cependant, à chaque modification, les black hats révisent à leur tour leurs pratiques, ce qui oblige les concepteurs à réviser à nouveau l'algorithme, etc.

Si nous représentons schématiquement les principales modifications algorithmiques - dont les noms sont Boston, Cassandra, Caffeine, Panda, Pingouin... -, nous obtenons un " graphe sociotechnique ${ }^{39}$, où nous voyons schématiquement comment Google, pour continuer à associer un maximum d'acteurs à son " programme d'action ${ }^{40}$ et promouvoir une norme de publication qui lui convient, fait subir plusieurs détours à l'algorithme. À chaque changement, le dispositif devient un nouveau dispositif, qui se substitue à l'ancien (axe vertical : OU) pour maintenir l'association de Google à ses partenaires white hats et éventuellement en rallier de nouveaux (axe horizontal : ET). La ligne oblique tracée sur le schéma et reliant l'ensemble des révisions algorithmiques désigne la dynamique normative qui influence à la fois les pratiques de publications et les critères algorithmiques. Celle-ci est sans cesse renégociée étant donné les actions des black hats, qui n'écoutent que leurs propres intérêts et agissent de manière non-éthique (c'est-à-dire ils agissent en fonction de ce qu'ils savent du faire de Google et d'une façon qui, si elle se généralisait, mettrait en péril l'existence du moteur), et les white hats, qui se conforment aux recommandations de Google et agissent conformément à un impératif catégorique kantien.

Un élément mérite enfin d'être relevé concernant la négociation d'une norme de publication : le recours à la délation. Dans ses recommandations, Google invite les éditeurs à dénoncer ceux qui, parmi eux, ne respectent pas les règles édictées : " Si vous constatez qu'un site participe à des systèmes de liens visant à manipuler le classement PageRank, veuillez nous en informer. Nous utiliserons ces informations pour améliorer nos algorithmes de détection de ces liens ${ }^{41}$. Il y a donc une possibilité d'autorégulation de la part des éditeurs, qui se surveillent les uns les autres et désignent celui qui doit être puni car il a manqué à la règle édictée non pas par eux mais par l'entreprise Google qui, seule, peut effectivement punir celui qui a été dénoncé et qui, seule, a le pouvoir d'édicter les règles, mais qui ne peut pas pour autant obliger les éditeurs à agir de telle ou telle façon.

38. Pour une liste complète des changements algorithmiques effectués (et communiqués au public) : " L'algorithme Google et ses versions évolutives depuis 2000 ", Seolius, [en ligne] https://www.seolius.com/outils/google-algorithmes, consulté le 15 février.

39. Voir : Bruno Latour, Philippe Mauguin \& Geneviève Teil, « Une méthode nouvelle de suivi des innovations : le graphe socio-technique ", in Dominique Vinck (dir.), La Gestion de la recherche. Nouveaux problèmes, nouveaux outils, Bruxelles, De Boeck, 1991, p. 419-567.

40. Le programme d'action se veut programme d'action « qui se veut prédétermination des mises en scène que les [éditeurs] sont appelés à imaginer à partir du dispositif technique et des prescriptions (notices, contrats, conseils) » (cf. Madeleine Akrich, "Comment décrire les objets techniques ? , Techniques \& Culture, 9, 1987, p. 205-219). Il est une "série d'objectifs, d'états et d'intentions qu'un agent peut parcourir » (cf. Bruno Latour Pandora's Hope. Essays on the Reality of Science Studies, Cambridge MA/London, Havard University Press, 1999, p. 178).

41. Google, " Systèmes de liens ", Aide Search Console, [en ligne] https://support.google.com/webmasters/answer/66356, consulté le 15 février 2016. 


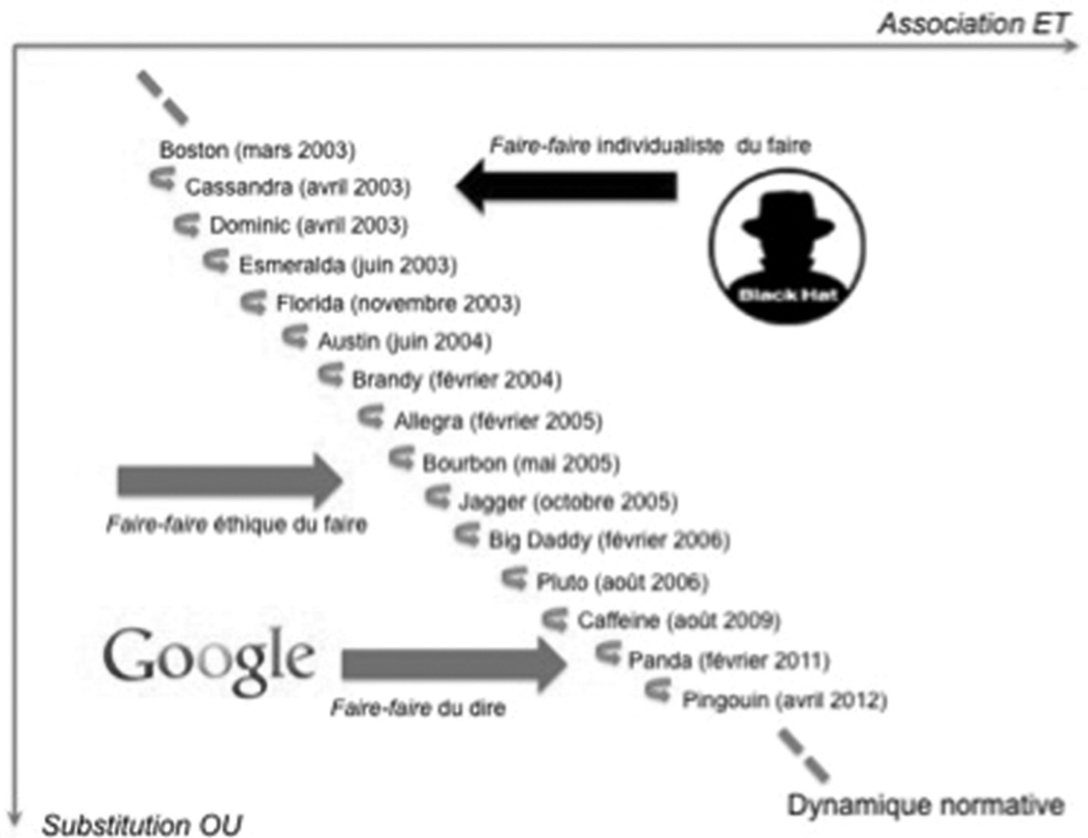

Figure 2. Graphe sociotechnique : modifications d'algorithme (2003-2012)

\section{Conclusion}

Reprenons notre argument de manière schématique. Le moteur fait quelque chose. Il y a un faire. Il consulte les documents, les indexe et répond à la requête d'un internaute en les triant. Étant donné sa position de quasi-monopole, ce faire de Google fait faire des actions aux éditeurs : c'est ce que nous avons appelé «le faire-faire du faire ». Il y a deux formes de ce faire-faire : un faire-faire éthique et un faire-faire individualiste. Le faire-faire éthique va dans le sens des intérêts de Google, des utilisateurs du moteur et de tous les autres éditeurs puisqu'il ne met pas en péril le dispositif qui leur permet d'interagir, alors que le faire-faire individualiste va dans le sens des seuls intérêts de celui qui le pratique. Si les concepteurs ne dévoilent pas le détail des critères algorithmiques, c'est en grande partie pour éviter que l'influence de ce faire-faire individualiste du faire ne soit trop importante, car cela risquerait de réduire la qualité du moteur et pourrait même l'empêcher de fonctionner. C'est également pour cette raison que les concepteurs du moteur disent aux éditeurs ce qu'il faut faire pour avoir un comportement éthique, en communiquant sur des blogs et des centres d'aide, dans le but que le faire-faire de ce dire, ajouté à la volonté de certains éditeurs d'adopter un comportement éthique, puisse contrebalancer les incitations à se comporter de façon malveillante.

Finalement, l'influence de Google sur le Web est le fruit d'une mise en tension entre le faire-faire éthique du faire, doublé par le faire-faire du dire, et le faire-faire individualiste du faire. Cette mise en tension s'apparente à une dynamique normative, dès lors qu'elle contribue à influencer les pratiques de tous les éditeurs concernés par l'action de Google, c'est-à-dire de presque tous les éditeurs du Web. 


\section{\begin{tabular}{l|l} 
Questionner les pouvoirs éditoriaux de Google & 103
\end{tabular}}

Nous avons pris l'exemple de l'influence exercée sur l'usage des liens hypertextes, mais il ne s'agit que d'un élément parmi tous les éléments susceptibles d'être influencés par Google. À bien y regarder, il semblerait que les enjeux liés au référencement puissent influencer l'ensemble des aspects de la production de contenus : le choix des sujets traités, le choix des formats, le choix des mots employés, la structure des sites, les fonctionnalités, la taille des images, la disposition des pages ${ }^{42}$.

Du point de vue méthodologique, il nous semble important de souligner ici qu'à notre avis, le pouvoir de faire faire ne peut pas être compris si l'on s'en tient, comme Theo Röhle, à l'observation de la stratégie de communication mise en place par un acteur comme Google pour dire aux éditeurs ce qu'ils doivent faire. Il s'agit aussi de considérer que les infrastructures physiques et les systèmes logiques agissent et font agir, disent et font dire. La réalité de ce que sont finalement les pratiques sur le Web et leurs résultats résulte de la tension entre le faire-faire du dire des concepteurs et le faire-faire du faire de ce qui a été conçu. Quand un acteur réussit à orienter cette tension à son avantage, la forme de pouvoir qu'il exerce s'apparente à une "gouvernementalité algorithmique ${ }^{43}$ : les actions des autres acteurs sont influencées par l'intermédiaire à la fois des algorithmes et du discours à propos de ces algorithmes sans jamais être forcés à rien.

Nous pensons que sur le Web les allers-retours entre les différents niveaux de performativité, semblables à ceux que nous avons décrits ici, sont monnaie courante. Et nous pensons qu'il est impossible de comprendre les tenants et les aboutissants normatifs des rapports de pouvoir sans considérer le rôle joué par l'infrastructure physique (hardware) et la couche logicielle (software) au sein des chaînes de médiations, dès lors que ceux-ci peuvent susciter des effets non voulus par les concepteurs et les utilisateurs des technologies numériques. Puisque le pouvoir est sur le Web comme ailleurs, selon les termes de Michel Foucault, un " faisceau de relations », alors il est du ressort — et du devoir — des sciences de l'information et de la communication de problématiser et de déplier analytiquement ce faisceau sans omettre le rôle qu'y jouent la technique en général et les algorithmes en particulier.

\section{Bibliographie}

Andrieu Olivier, Réussir son référencement Web. Stratégie et techniques SEO, Paris, Eyrolles, 2013

Appel Violaine, Boulanger Hélène \& Massou Luc, Les dispositifs d’information et de communication. Concepts, usages et objets, Bruxelles, De Boeck, 2010, 246 p.

42. Voir : Guillaume Sire, Les moteurs de recherche, op. cit.

43. À propos de ce terme, voir : Antoinette Rouvroy, " Gouvernementalité algorithmique et perspectives d'émancipation : le disparate comme condition de l'individuation par la relation ? ", Réseaux, 177, 2013, p. 163-196. Voir aussi : Romain Badouard, Clément Mabi \& Guillaume Sire, « Inciter, contraindre, encadrer. Trois régimes de gouvernementalité numérique », Working paper présenté dans le séminaire du réseau DEL, 12 février 2015, [en ligne] https://guillaumesire.wordpress.com/publicationscommunications/, consulté le 15 février 2016. 
AT Internet, Baromètre des moteurs de recherche, 2014, [en ligne] http://www.atinternet.com/ressources/ressource/barometre-des-moteurs-de-recherchenovembre-2014/, consulté le 30 janvier 2016

Badouard Romain, Mabi Clément \& Sire Guillaume, "Inciter, contraindre, encadrer. Trois régimes de gouvernementalité numérique ", Working paper présenté dans le séminaire du réseau DEL, 12 février 2015, [en ligne] https://guillaumesire.wordpress.com/publications-communications/, consulté le 15 février 2016

Battelle John, The search : how Google and its rivals rewrote the rules of business and transformed our culture, New York, Nicholas Brealey Publishing, 2005, 288 p.

Bonaccorsi Julia, Julliard Virginie, "Dispositifs de communication numériques et médiation du politique. Le cas du site Web d'Ideal-Eu », in Aghababaie Mona, Bonjour Audrey, Clerc Adeline \& Rauscher Guillaume (dir.), Usages et enjeux des dispositifs de médiation, Nancy, Presses universitaires de Nancy, 2010, p. 65-78

Boutet Charles-Victor, Ben Amor Samy, «Vers l'active SEO 2.0 », Les Cahiers du numérique, 6, 2010, p. 179-198

Brin Sergey, Page Larry, «The Anatomy of a Large-Scale Hypertextual Web Search Engine ", Computer Science Department, Stanford University, 1998

Cardon Dominique, La démocratie Internet. Promesses et limites, Paris, Seuil, 2010, 102 p.

Cardon Dominique, « Dans l'esprit du PageRank. Une enquête sur l'algorithme de Google ", Réseaux, 177, 2013

De Certeau Michel, L'invention du quotidien, 1. Arts de faire, Paris, UGE/10-18, 1980, 348 p.

Foucault Michel, Surveiller et Punir, Paris, Gallimard, 1975, p. 35

Foucault Michel, "Le jeu de Michel Foucault », Ornicar ? Bulletin Périodique du champ freudien, 10, 1977, p. 62-93

Foucault Michel, Dits et écrits, Tomes 1 \& 2, Paris, Gallimard, 1994

Google, "Consignes aux webmasters ", Aide Search Console, [en ligne] https://support.google.com/webmasters/answer/35769?hl=fr\&ref_topic=6002025, consulté le 15 février 2016

Google, "Systèmes de liens ", Aide Search Console, [en ligne] https://support.google.com/webmasters/answer/66356, consulté le 15 février 2016

Hindman Matthew, Tsioutsiouliklis Kostas \& Johnson Judy A., " "Googlearchy" : How a Few Heavily Linked Sites Dominate Politics on the Web », Annual meeting of the Midwest Political Science Association, 2003

Introna Lucas D. \& Nissenbaum Helen, " Shaping the Web : why the politics of search engines matters ", The Information Society, 16, 2000, p. 169-185

Latour Bruno, Pandora's Hope. Essays on the Reality of Science Studies, Cambridge MA/London, Havard University Press, 1999, p. 178

Latour Bruno, Changer la société, refaire de la sociologie, Paris, La Découverte, 2007a, 400 p.

Latour Bruno, "Une sociologie sans objet ? Remarques sur l'interobjectivité » in Debary Octave \& Turgeon Laurier, Objets et mémoires, Paris, Maison des Sciences de l'Homme, 2007b, p. 37-58

Latour Bruno, Mauguin Philippe \& Teil Geneviève, «Une méthode nouvelle de suivi des innovations : le graphe socio-technique ", in Vinck Dominique (dir.), La Gestion de la recherche. Nouveaux problèmes, nouveaux outils, Bruxelles, De Boeck, 1991, p. 419-567

Mabi Clément, " Les conceptions de la participation citoyenne inscrites au sein d'un dispositif de concertation ", Journées doctorales GIS Participation, 2011, [en ligne] http://www.participation-et-democratie.fr/content/les-conceptions-dela-participation-citoyenne-inscrites-au-sein-dun-dispositif-de-concertati, consulté le 15 février 2016 


\section{\begin{tabular}{l|l} 
Questionner les pouvoirs éditoriaux de Google 105
\end{tabular}}

Massit-Folléa Françoise, Méadel Cécile \& Monnoyer-Smith Laurence, Normative Experience in Internet Politics, Paris, Presse des Mines, 2012, 266 p.

Mathias Paul, Qu'est-ce que l'Internet ?, Paris, Vrin, coll. «Chemins Philosophiques », 2009

Monnoyer-Smith Laurence, "Le Web comme dispositif : comment appréhender le complexe? » in Barats Christine (dir.), Manuel d'analyse du Web en Sciences Humaines et Sociales, Paris, Armand Colin, 2013

Moskwa Susan, « Reorganizing internal vs. external backlinks ", Google Webmaster Central Blog, 31 août 2011, [en ligne] http://googlewebmastercentral.blogspot.fr/2011/08/reorganizing-internal-vsexternal.html, consulté le 15 février 2016.

Page Larry, «Method for Node Ranking in a Hyperlinked Database », U.S. Patent 6,285,999, filed January 9, 1998, and issued September 4, 2001

Page Larry, « Method for Scoring Documents in a Linked Database », U.S. Patent 6,799,176, filed July 6, 2001, and issued September 28, 2004

Page Larry, Brin Sergey, Motwani Rajeev \& Winograd Terry, «The PageRank Citation Ranking: Bringing Order to the Web », Technical Report, Stanford InfoLab, 1999

Pandurangan Gopal, Raghavan Prabhakar \& Upfal Eli, «Using PageRank to characterize Web structure », Internet Mathematics, 3, 2006, p. 1-20

Richard Raphaël, Google décodé : les 300 critères de classement. Tomes 1 \& 2, Webmarketing Academy, 2011

Rieder Bernhard, «What is in PageRank? A Historical and Conceptual Investigation of a Recursive Status Index », Computational Culture, 2012

Rieder Bernhard, Sire Guillaume, "Conflicts of Interest and Incentives to Bias: A Microeconomic Critique of Google's Tangled Position on the Web ", New Media \& Society, 16, 2014 p. 195-211

Röhle Theo, «Dissecting the Gatekeepers. Relational Perspectives on the Power of Search Engines ", in Becker Konrad \& Stalder Felix (dir.) Deep Search. The Politics of Search beyond Google, Innsbruck, StudienVerlag, 2009, p. 117-132.

Rouvroy Antoinette, «Gouvernementalité algorithmique et perspectives d'émancipation : le disparate comme condition de l'individuation par la relation ? ", Réseaux, 177, 2013, p. 163-196.

SEOlius, «L'algorithme Google et ses versions évolutives depuis 2000 », Seolius, [en ligne] https://www.seolius.com/outils/google-algorithmes, consulté le 15 février 2016.

Sire Guillaume, Google, la presse et les journalistes. Analyse interdisciplinaire d'une situation de coopétition, Bruxelles, Institut du Droit de la Concurrence/Bruylant, coll. «Sciences Politiques », 2015, $426 \mathrm{p}$.

Sire Guillaume, Les moteurs de recherche, Paris, La Découverte, coll. "Repères », 2016, $128 \mathrm{p}$.

Sire Guillaume, Rieder Bernhard, « Dans les ramures de l'arbre hypertexte, Analyse des incitations générées par l'opacité du moteur Google ", French Journal for Media Research, 3, 2015, [en ligne] http://frenchjournalformediaresearch.com/lodel/docannexe/file/468/arbre_de_ navigation.pdf, consulté le 15 février 2016

Van Couvering Elizabeth, «The History of the Internet Search Engine: Navigational Media and the Traffic Commodity ", in Zimmer Michael \& Spink Amanda (dir), Web Search. Multidisciplinary Perspectives, Berlin, Springer, 2008, p. 177-206

\section{GUILLAUME SIRE}


